\title{
349 - Cognitive-Behavioral Therapy for Caregiving Relatives of People with Dementia: Three- Year Follow-Up of a Randomized Controlled Trial
}

Mareike C. Sittler, Nils F. Töpfer, Franziska Meichsner, Christina Theurer, \& Gabriele Wilz

Background: Even if cognitive-behavioral therapy (CBT) for caregiving relatives of people with dementia (PwD) has been found to be effective across various outcome measures, investigations into the longterm effects of these interventions remain scarce. Therefore, the purpose of the present study was the evaluation of a CBT intervention for caregiving relatives of PwD three years after intervention onset.

Method: 273 caregivers were randomly assigned to receive the intervention (IG) or usual care (CG). IG participants received over 6 month twelve 50-min sessions of individual CBT by trained psychotherapists. Symptoms of depression (CES-D), physical health symptoms (GBB-24), challenging behavior (BEHAVE-AD), quality of life (WHOQOL-BREF), utilization of psychosocial resources (RES), burden of care, coping with the care situation, and emotional well-being (visual analogue scales) were assessed three years after baseline. Data were analyzed using generalized ANCOVA.

Results: 164 participants (IG: $n=83$, CG: $n=81 ; 60 \%$ of the baseline sample) participated in the threeyear follow-up. Based on changes in the caregiving situation at three-year follow-up, we divided the sample into three subgroups: "still caring at home" $(n=52)$, "nursing home placement of the care recipient" $(n=29)$, "bereaved caregivers" $(n=83)$. Positive effects were found in the subgroup of caregivers "still caring at home" (on burden of care, coping with challenging behavior, social relationships QoL-domain) and "bereaved caregivers" (on overall QoL and physical health QoL-domain) compared to the CG. However, IG participants who decided for "nursing home placement of the care recipient" had poorer outcomes on a few measures (overall QoL, psychological health, emotional wellbeing, utilization of resources related to well-being).

Discussion: It is impressive that CBT for caregiving relatives yielded positive effects in caregivers still caring at home and bereaved caregivers investigating long-term effects three years after baseline. Many challenges arise over the course of the different caregiving trajectories with changes in the caregiving situation being probably particularly influential. We will provide some ideas on how effects could be further sustained and discuss the need for further investigating the impact of changes in the caregiving situation. 